\title{
A Multi-sensor Approach for Grasping and 3D Interaction ${ }^{1}$
}

\author{
Zhiyong Huang ${ }^{a}$ \\ Ronan Boulica \\ Nadia Magnenat Thalmann ${ }^{b}$ \\ Daniel Thalmann ${ }^{\mathrm{a}}$ \\ a) LIG - Computer Graphics Lab \\ Swiss Federal Institute of Technology \\ Lausanne, CH-1015 Switzerland \\ fax: +41-21-693-5328 \\ email: \{huang; boulic; thalmann\}@di.epfl.ch \\ b) MIRALab-CUI, University of Geneva \\ 24 rue du Général Dufour \\ Geneva, CH -1211 Switzerland \\ fax: +41-22-320-2927 \\ email: thalmann@cui.unige.ch
}

\begin{abstract}
In this paper, we propose a multi-sensor based method of automatic grasping motion control for multiple synthetic actors. Despite the fact that it is described and implemented in our specific model, the method is general and can be applicable to other models. A heuristic method is defined to decide the different grasping strategies from object geometry, hand geometry and observation of real grasping. Inverse kinematics can derive the final posture of the arms in order to bring the hands around the object. Multi-sensor object detection decides the finger contact points on the object and determine their position and orientation. Then, a group of polynomials derived from Euler-Lagrange equation is used to interpolate between the initial and final arm postures resulting in a more realistic real-time motion than linear interpolation. We also present $3 \mathrm{D}$ interactive grasping examples involving multiple synthetic actors.
\end{abstract}

Keywords: synthetic actor, grasping, DataGlove, multi-sensors, inverse kinematics

\section{Introduction}

Research in human grasping is an important field in medical study and robotics. With the advents of synthetic actors in computer animation, it has also become a key issue in this field. Magnenat Thalmann et al. (1988) proposed a semi-automatic way of grasping for a synthetic actor interacting with the environment. They also describe hand deformations based on the concept of joint-dependent local deformation operators. A knowledge-based approach is suitable for simulating human grasping, and an expert system can be used for this purpose (Rijpkema and Girard 1991). Kunii et al. (1993) have presented a model of hands and arms based on manifold mappings. They have also considered dependencies between joints and made some realistic animation of hand gesture. Recently, Mas and Thalmann [Mas and Thalmann 94] have presented a hand control and automatic grasping system using an inverse kinematics based method. The work described in the present paper is based on this earlier work and extends it in three ways:

\footnotetext{
${ }^{1}$ published in Proc. CGI ‘95, Academic Press
} 
1) A generalization of the concept of multi-sensors for hand joints is proposed to allow synthetic actors to grasp common objects, share them and exchange them with other actors.

2) In order to produce a more realistic arm motion, polynomials based on the Lagrange-Euler equation have been introduced.

3) 3D interactive grasping method based on DataGlove and head-mounted displays has been added for VR applications

Finally, it is important to mention that all the methods described in this paper have been integrated into TRACK (Boulic et al. 1994b), our general motion control system, a key component in the ESPRIT HUMANOID project.

In the following sections, we will first introduce our human model, especially on hand and arm in Section 2. Then the grasping motion control is presented in Section 3 emphasizing the multi-sensor approach. The polynomials from Lagrange-Euler equation are deduced and discussed in Section 4. The 3D interactive grasping approach is described in Section 5. Finally we present some results, evaluate our method, and discuss the future work.

\section{Modeling}

Our hand model is part of the HUMANOID data structure, which provides a complete human skeleton structure with rigid articulated figures connected by one to three DOFs at each joint. Special care has been given to the mobility of the hand model, due to the great complexity of the wrist-palm region on which the fingers articulate themselves. First at the level of the metacarpus, a small flexing mobility has been provided to model the deformations of the palm. Then a standard structure is used for the four following fingers: pinky, ring, middle and index. It is organized as:

- the first flexing DOF for closing the first knuckle;

- a pivoting rotation for the lateral mobility of the finger;

- the two final flexing DOFs for closing the corresponding knuckles.

The thumb has a very specific organization due to its special mobility:

- a pivoting rotation around an axis oriented like the index finger vector;

- a twisting rotation along the thumb axis naturally coupled with the previous one so as to control the orientation of the thumb finger;

- a lateral rotation perpendicular to the twisting and the natural flexion axis (abduct);

- three successive flexing DOFs for closing the following segments.

Figure 1 shows the degrees-of-freedom for the hand model and Figure 2 shows a view of the right hand skeleton and Fig. 3 a hand with attached volumes. 


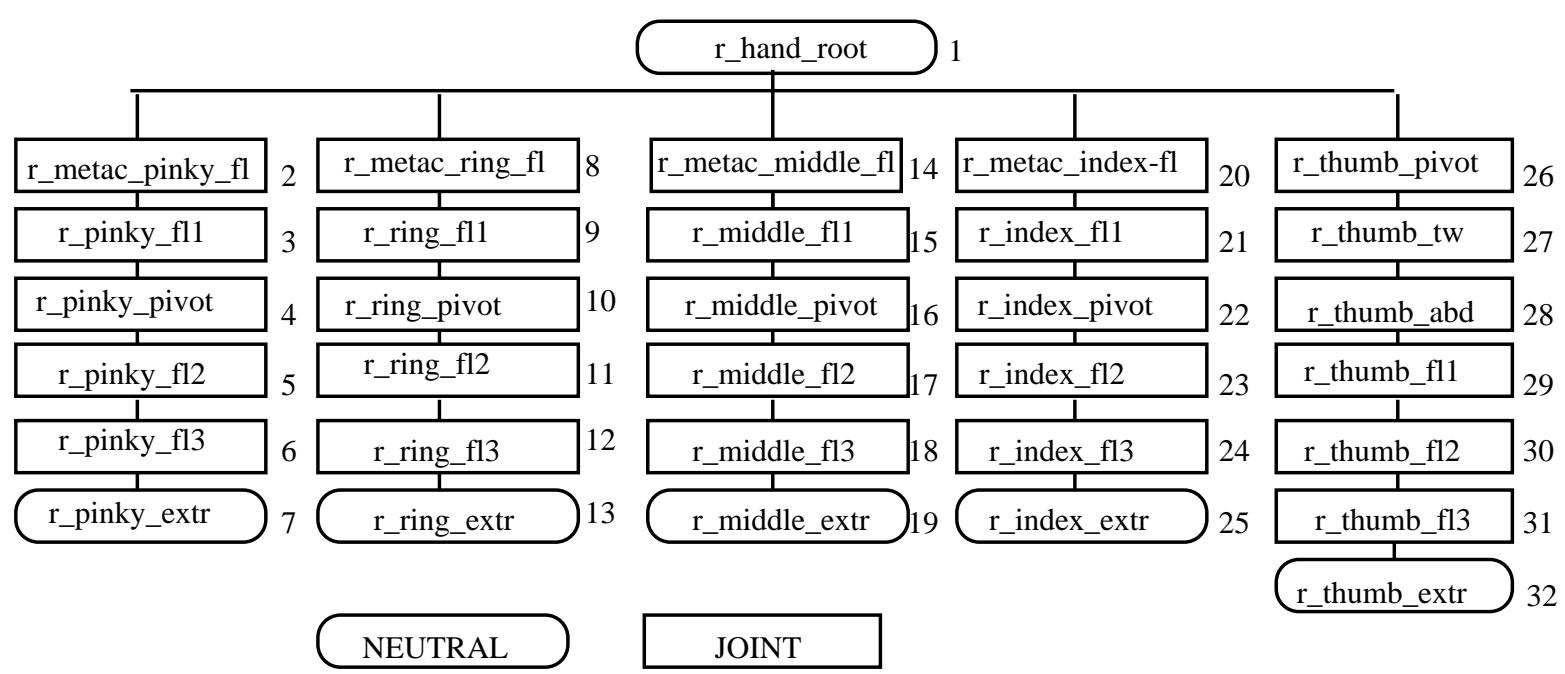

Figure 1. The name table of DOFs of hand model

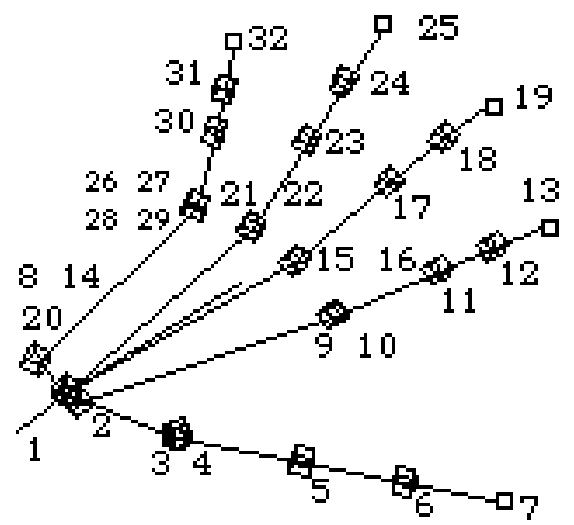

Figure 2. 3D view of the skeleton of the right hand indicating the location of the node_3D in the rest position. (the integer values refer to Figure 1).

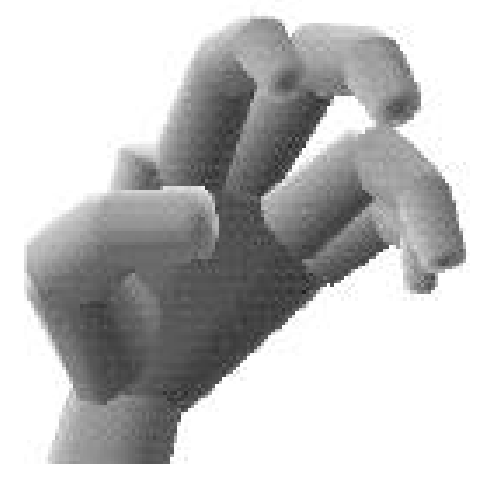

Figure 3. Hand attached with volumes for grasping

\subsection{Arm model}

For grasping process, the arm is also an essential part and we propose to model it using the following DOFs (see Figure 4):

At the clavicle :

- clav_abduct : up and down motion in the coronal plane (y BODY )

- clav_rotate : rotation in the transverse plane (z BODY ) 
At the scapula :

- scap_abduct : up and down motion in the coronal plane (y BODY )

- scap_rotate : rotation in the transverse plane (z BODY)

At the shoulder :

- shoulder_flexion : forward-backward motion in the sagital plane (x BODY )

- shoulder_abduct : side motion in the coronal plane (y BODY )

- shoulder_twisting : along the forearm axis

At the elbow :

- elbow_flexion : flexion-extension of the arm in the sagital plane (x BODY)

- elbow_twisting : along the arm axis.

At the wrist :

- wrist_flexion : rotation of the hand in the coronal plane (y BODY)

- wrist_pivot : rotation of the hand in the sagital planes (x BODY)
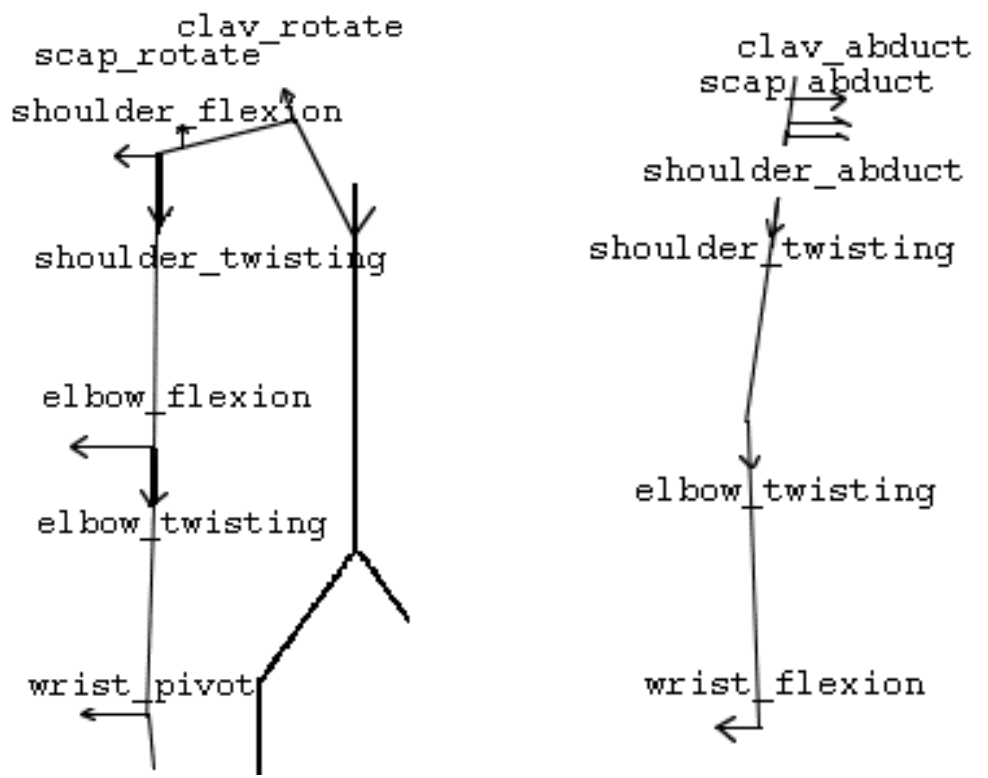

Figure 4. The front and side views of the mobility of the arm in rest position.

\section{Grasping motion control}

There are three steps in this procedure:

- inverse kinematics to find the final arm posture

- grasping decision

- multi-sensor process

\subsection{Inverse kinematics to find the final arm posture}

Inverse kinematics (Philips et al. 1990) is a widely used technique in Robotics. This technique is suited to control or constraint strategic parts of the articulated figure which location depends on a set of parameters. The control scheme is based on a linearization of the location problem at the current state of the system.

Before using inverse kinematics, the first problem is to select an end effector on the open chain of the articulated figure. There are many choices for grasping that result in different treatments. As shown in Figure 5, we choose the hand center as the end effector for convenience. The hand center can be on palm surface or above it. It depends 
whether the final frames are inside or not. The $\mathrm{Z}$ axis is perpendicular to the palm of the hand, $X$ is parallel to the direction of straight fingers, and $Y$ is the cross product of $X$ and $\mathrm{Z}$. The frame should be aligned to the final object frame for the final posture. This final object frame depends on the geometry of the object, the hand size, and avoidance of collision if more than one hand are grasping one object. Our heuristic approach is discussed in the next section.

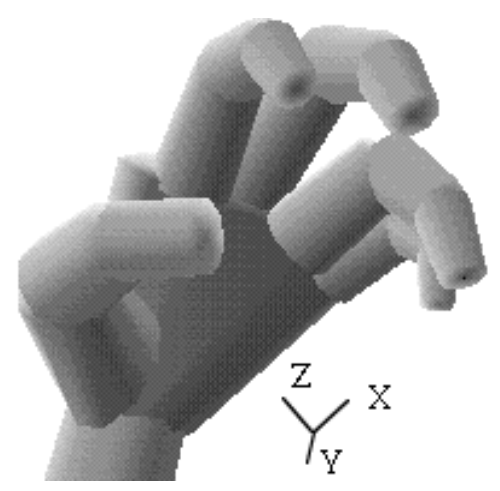

Figure 5. The hand center frame used as end effector for inverse kinematics

\subsection{Heuristic grasping decision}

From observation on real grasping and methods in robotics (see (Mas and Thalmann 1994), there are many ways of grasping with one or two hands, with two to five fingers of each hand according to the type, geometry and weight of the object to be grasped. We summarize it in Table 1 only for solid primitives. For a more general object, the decision should be made according to its multiple bounding volumes. The bounding volumes can be a sphere, a cube, a cylinder etc. For example, for the surface model of a human body, the head will be bounded by a sphere, and the neck by a cylinder.

\begin{tabular}{|c|l|c|}
\hline Type & Size & Grasping way \\
\hline \multirow{2}{*}{ Cube } & thin & lateral \\
\cline { 2 - 3 } Sphere & thick & pinch \\
\cline { 2 - 3 } & small diameter & tripod \\
\hline $\begin{array}{c}\text { Cylinder } \\
\text { and } \\
\text { Frustum }\end{array}$ & large diameter & wrap \\
\cline { 2 - 3 } & small diameter & pinch \\
\hline
\end{tabular}

Table 1. The grasping decision

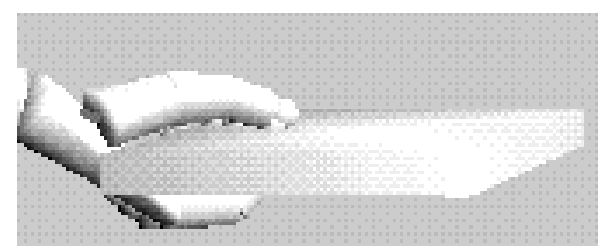

a. cube lateral

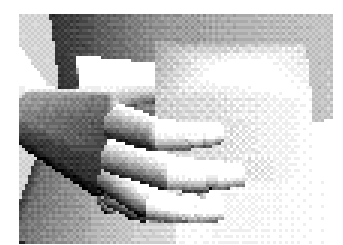

b. cube pinch 


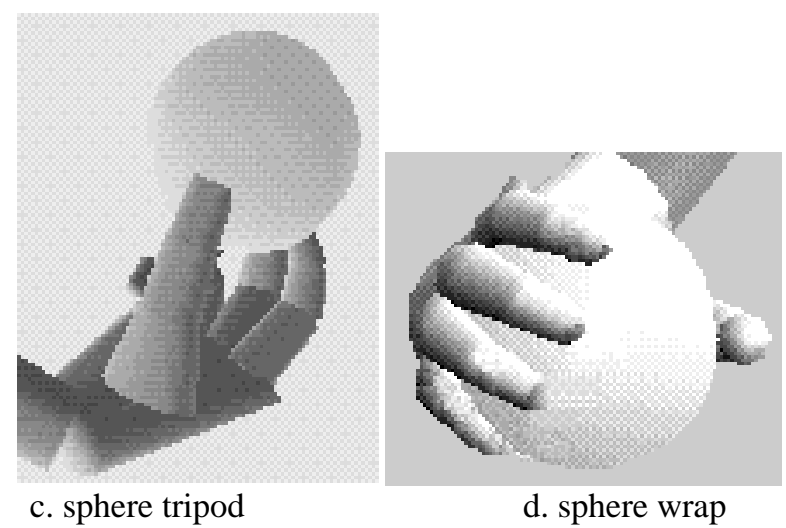

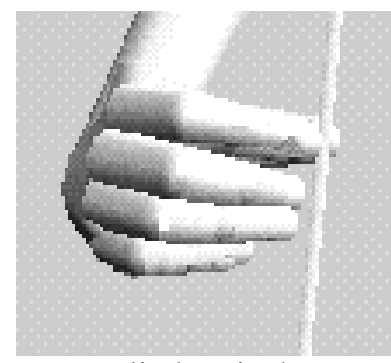

e. cylinder pinch

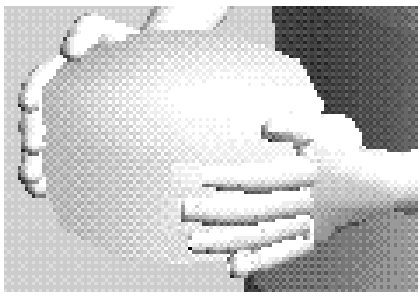

f. cylinder wrap

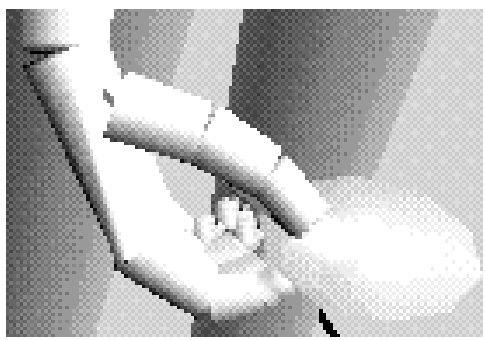

g. cylinder wrap

Figure 6. The different grasping ways for different objects

In all cases, pinch is applied if the primitive is too small to be grasped by more than two fingers. Two hands are applied if it is too large to be taken by one hand. Small and large are compared with hand size.

Now, we come back to the problem of finding our final object frame. For the cube, this is done as follows:

- we state that the thumb always remain on a visible surface;

- we prevent the palm face the exterior side of the body because it results in an unnatural grasping;

- the contact faces can be chosen by minimizing a weighted sum of translation and rotation between the initial and final hand postures (Mas and Thalmann 1994).

The goal cube frame is then determined according to the following procedure:

- set the center of the cube as the initial origin of the axis;

- transform the cube to hand local frame to check whether it collides with hand at initial posture;

- until no collision has been found;

- otherwise the origin should be moved closer to the border of cube and making collision detection again ;

- the orientation of each axis is the same as the local frame of cube;

Sphere, cylinder and frustum frame is similar to cube, all starting from the center of object with orientation the same as their local frame. For large radius one, the collision detection will move the origin to its surface. Fig. 6 shows examples.

For a general free form surface object, the origin of object frame can start from one point inside the object which may be the center of its bounding object (either a sphere, cube or cylinder according to its shape). In Figure 7, some final frames resulting from this method are displayed. For the head, it starts from the bounding cylinder of the neck. The collision detection should be performed on the object. 

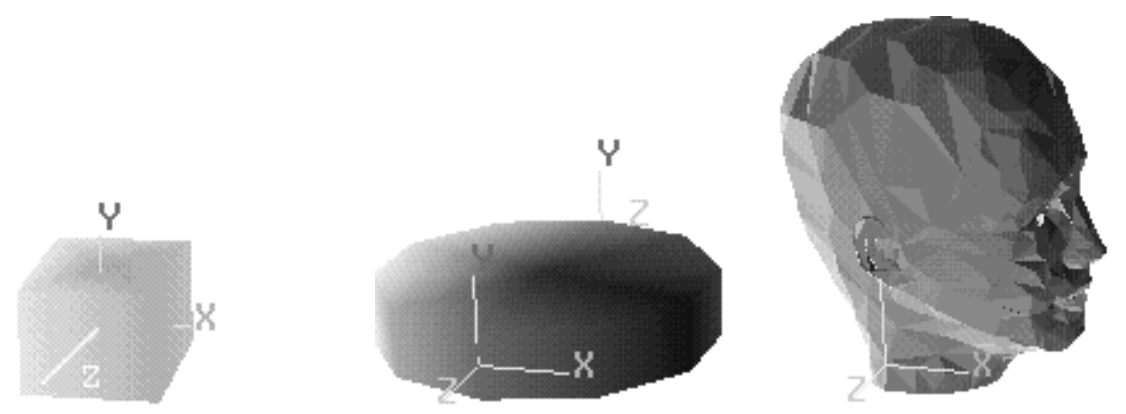

Figure 7. The final grasping frame from heuristic way

\subsection{Multi-sensor hand}

In this section, we present a multi-sensor approach for the grasping. Our approach is adapted from the use of proximity sensors in Robotics (Espiau and Boulic 1985), the sensor-actuator networks (van de Panne and Fiume 1993) and recent work on human grasping (Mass and Thalmann 1994). In our work, the sphere multi-sensors have both touch and length sensor properties, and have been found very efficient for synthetic actor grasping problem.

Multi-sensors are considered as a group of objects attached to the articulated figure. A sensor is activated for any collision with other objects or sensors. Here we select sphere sensors for their efficiency in collision detection (Figure 8).

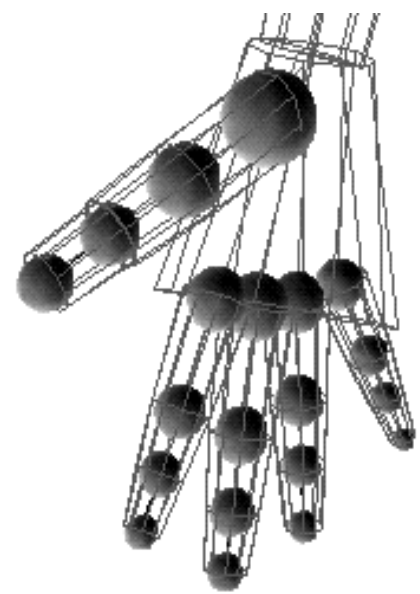

Figure 8 . The hand with sphere sensors at each joint

Each sphere sensor is fitted to its associated joint shape with different radii. This configuration is important in our method because when a sensor is activated in a finger, only the articulations above it stop moving, while others can still move. By doing this way, all the fingers are finally positioned naturally around the object, as shown in Figure 9. 


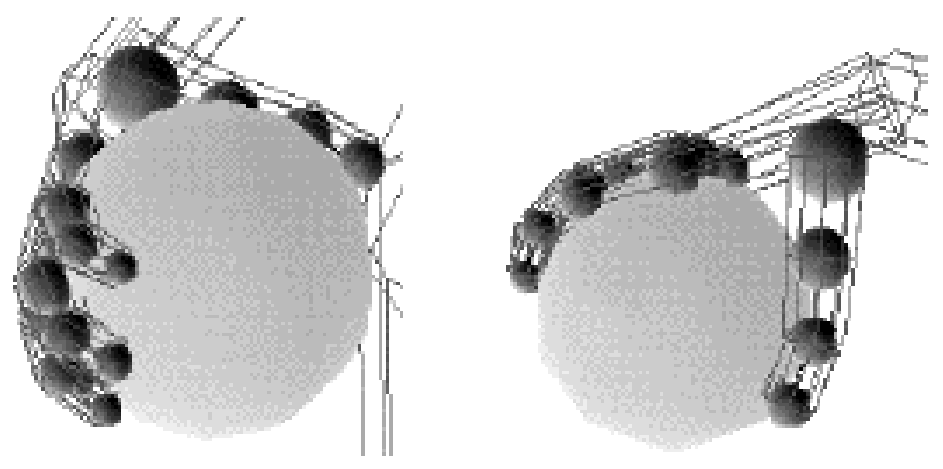

Figure 9. One example shown sensors in grasping

When grasping a free form surface object, the sphere sensors are detecting collision with the object. We do not discuss more on collision detection which is beyond the scope of this paper.

To summarize: after the hand center frame is aligned with the object frame, the fingers are closed according to the different strategies, e.g. pinch, wrap, lateral, etc., while sensor-object and sensor-sensor collisions are detected. When one sensor is activated, all articulations above it are blocked. The grasping is completed when the remaining sensors are activated or the joints reach their limit. The final arm moving problem is now discussed in the next section.

\section{Moving arm with polynomials based on Lagrange-Euler equation}

In this section, the problem to solve is to move the arm from its initial posture to the final one derived by inverse kinematics. We introduce polynomials which try to mimic the well known invariance of the velocity profile in single goal movements [Bullock and Grossberg 1988]. Based on Figure 9, we define some frames and angles used in formulae of arm dynamics.

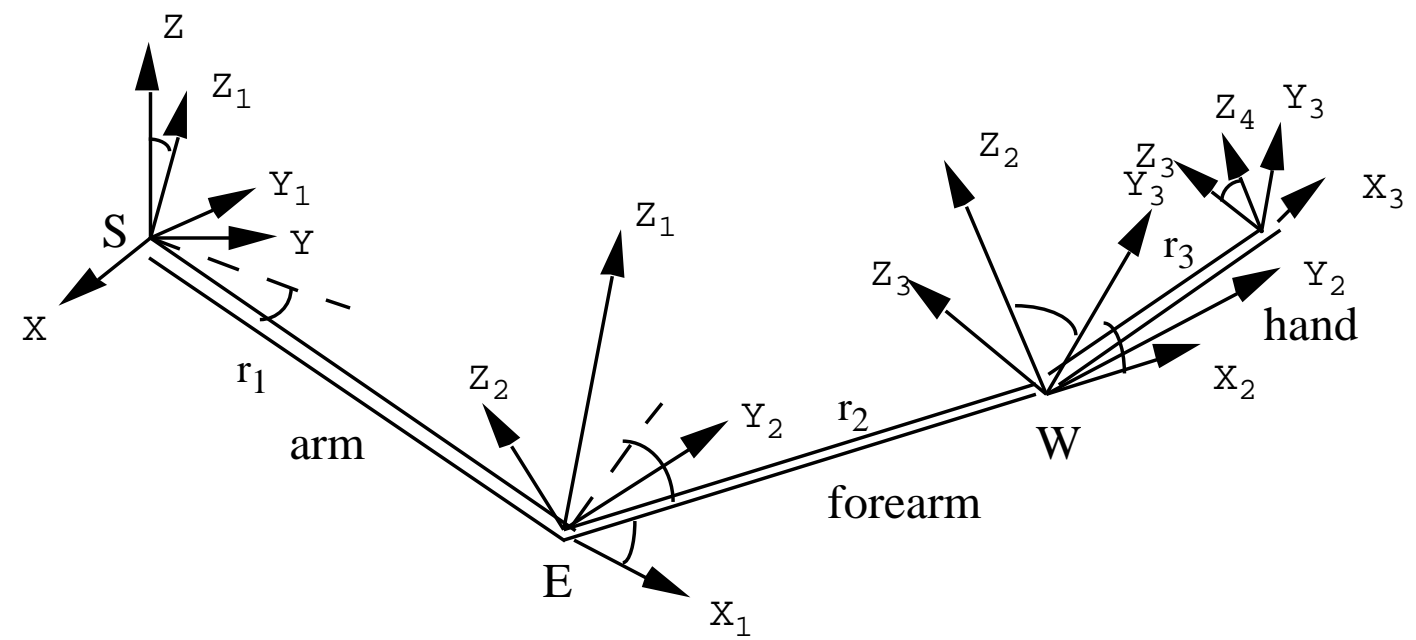

Figure 10. Seven angles of 3 piece arm used in formula

There are seven DOFs of our arm model. On top of each link, there is one local frame, $\mathrm{XYZ}, \mathrm{X}_{1} \mathrm{Y}_{1} \mathrm{Z}_{1}$ and $\mathrm{X}_{2} \mathrm{Y}_{2} \mathrm{Z}_{2}$. . Now we use spring and damping model at each joint to simulate the muscles.

- $\quad$ spring torque is of the form $\vec{M}_{\theta_{i}}^{\text {spring }}=k_{\theta_{i}}(t) \theta_{i} \vec{e}, \quad i=1, \cdots, 7$

- damp torque is of the form $\vec{M}_{\theta_{i}}^{\operatorname{damp}}=h_{\theta_{i}} \dot{\theta}_{i} \vec{e}, \quad i=1, \cdots, 7$ 
- gravitation field is of the form

- total energy of arm is $E=\int_{t_{0}}^{t_{1}} d t P\left(\theta_{1}, \cdots, \theta_{7}, \dot{\theta}_{1}, \cdots, \dot{\theta}_{7}, t\right)$

where $P$ is the total power resulting from spring, damp and gravity. $\theta_{i}=\theta_{i-\text { goal }}-\theta_{i-\text { current }}$ with $\theta_{i-\text { goal }}$ is the goal value and $\theta_{i-\text { current }}$ is the current value of joint $\mathrm{i}$. Time $\mathrm{t}$ changes from $\mathrm{t}_{0}$ to $\mathrm{t}_{1} . h_{\theta_{i}}$ and $k_{\theta_{i}}(t)$ are coefficient functions to be found.

- The power resulting from the sprint torque: $P_{\theta_{i}}^{\text {spring }}=\left|k_{\theta_{i}}(t)\right| \cdot \dot{\theta_{i}}, \quad i=1, \cdots, 7$

- Similarly the power from damping: $P_{\theta_{i}}^{\text {damp }}=h_{\theta_{i}} \cdot \dot{\theta}_{i}^{2}, \quad i=1, \cdots, 7$

- The power from gravitation: $P^{\text {gravity }}=\frac{d}{d t} \sum_{i=1}^{3} \vec{m}_{i} \vec{g} \cdot \vec{G}_{i}$, in Figure 10.

where $\vec{G}_{1}=0.5 \vec{r}_{1}, \vec{G}_{2}=\vec{r}_{1}+0.5 \vec{r}_{2}, \vec{G}_{3}=\vec{r}_{3}+\vec{r}_{2}+0.5 \vec{r}_{3}, \vec{r}_{1}, \vec{r}_{2}$ and $\overrightarrow{r_{3}}$ are arm vector

By use all these power terms into Lagrange-Euler equation,

$$
\frac{\partial P}{\partial \theta_{i}}-\frac{d}{d t} \frac{\partial P}{\partial \dot{\theta}_{i}}=0, \quad i=1, \cdots, 7(4.1)
$$

We will get:

$$
\frac{\partial P^{\text {gravity }}}{\partial \theta_{i}}-\frac{d}{d t} \frac{\partial P^{\text {gravity }}}{\partial \dot{\theta}_{i}}+\frac{\partial}{\partial \theta_{i}} \sum_{i}\left(h_{\theta_{i}} \dot{\theta}_{i}^{2}+\hat{k}_{\theta_{i}}(t) \dot{\theta}_{i}\right)-\frac{d}{d t}\left(\frac{\partial}{\partial \dot{\theta}_{i}} \sum_{i}\left(h_{\theta_{i}} \dot{\theta}_{i}^{2}+\hat{k}_{\theta_{i}}(t) \dot{\theta}_{i}\right)\right)=0
$$

where $\hat{k}_{\theta_{i}}(t)=\left|k_{\theta_{i}}(t)\right|$

Considering gravitation is conserved force, $\frac{\partial P^{\text {gravity }}}{\partial \theta_{i}}-\frac{d}{d t} \frac{\partial P^{\text {gravity }}}{\partial \dot{\theta}_{i}}$ is always 0 . By calculating the left part, that is $-\frac{d}{d t}\left(2 h_{\theta_{i}} \dot{\theta}_{i}+\hat{k}_{\theta_{i}}(t)\right)=0$. Finally we get,

$$
\dot{\hat{k}}_{\theta_{i}}(t)+2 h_{\theta_{i}} \ddot{\theta}_{i}(t)=0
$$

The next step is to decide the viscoelastic coefficient $k_{\theta_{i}}(t)$ to solve the equation (4.3). The arm is pushed away from the object before the final moment and it is pulled toward the object after the starting.

So $k_{\theta_{i}}(t)$ should have the form as in Figure 11, 


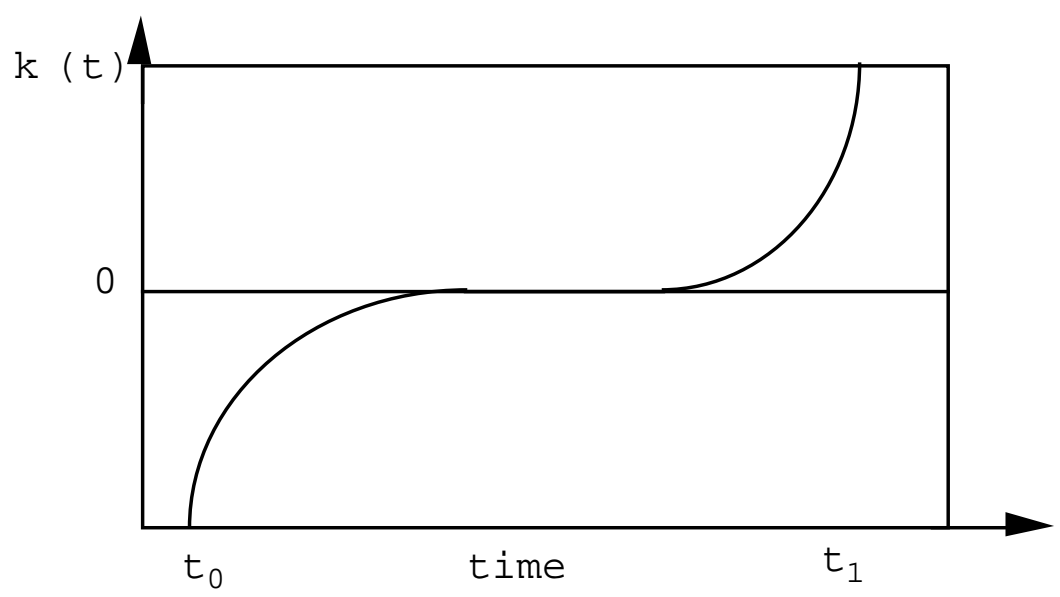

Figure 11. The expected shape of $k_{\theta_{i}}(t)$

Now we have the possibility to choose different polynomials (quadratic, cubic, etc.) to satisfy the above analysis. The simplest form is:

$$
k_{\theta_{i}}(t)= \begin{cases}-A h_{\theta_{i}}\left(t^{2}-t\right) & \text { for } t_{0} \leq t \leq 0.5\left(t_{1}-t_{0}\right) \\ +A h_{\theta_{i}}\left(t^{2}-t\right) & \text { for } t_{0} \leq t \leq 0.5\left(t_{1}-t_{0}\right)\end{cases}
$$

where A is a value determined from solving (4.3), typically a normal constant. Substituting (4.4) into (4.3), we obtain:

$$
\theta_{i}(t)=-6\left(\theta_{i}^{1}-\theta_{i}^{0}\right)\left[\frac{1}{3}\left(\frac{t-t_{0}}{t_{1}-t_{0}}\right)^{3}-\frac{1}{2}\left(\frac{t-t_{0}}{t_{1}-t_{0}}\right)^{2}\right\rfloor+\theta_{i}^{0}
$$

where $\theta_{i}^{0}$ and $\theta_{i}^{1}$ are the initial and final values of $\mathrm{i}$-th DOF.

$$
\theta_{i}(t)=-6\left(\theta_{i}^{1}-\theta_{i}^{0}\right)\left[\frac{1}{3}\left(\frac{t-t_{0}}{t_{1}-t_{0}}\right)^{3 n}-\frac{1}{2}\left(\frac{t-t_{0}}{t_{1}-t_{0}}\right)^{2 n}\right\rfloor+\theta_{i}^{0}
$$

If we replace power 3 (resp. power 2) by $3 n$ (resp. $2 n$ ), where $n$ is any positive integer, we can get a group of polynomials to satisfy the requirement.

In Figure 12, interpolation between initial and final postures are done with linear and polynomial method. The time sampling step is the same for both interpolations. We found that second one is closer to a real grasping. 


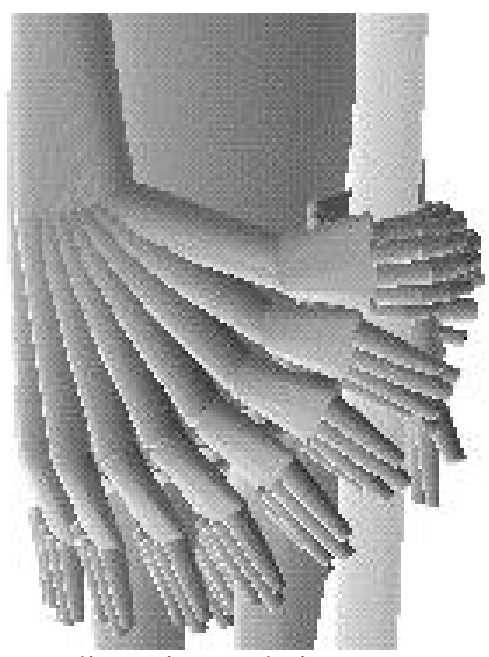

a. linear interpolation

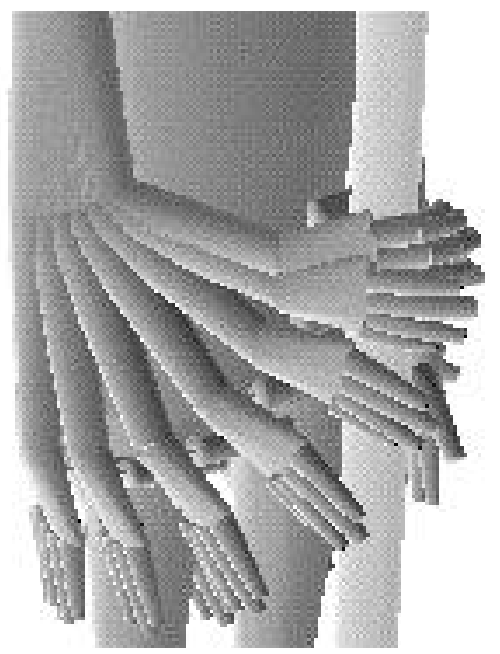

b. interpolation with polynomial $(n=1)$

Figure 12. With polynomial interpolation, the motion is accelerated and decelerated

\section{3D interactive grasping}

Three-dimensional interactive tools are widely available today, especially for Computer Graphics and virtual reality applications (Gobbetti et al. 1993). Among different 3D interactive devices, DataGlove ${ }^{\mathrm{R}}$ (or similar equipment) is the most interesting for grasping motion control. The Fifth Dimension Toolkit (Turner et al. 1990) provides facilities for using the DataGlove in applications as shown in Figure 13.

The IPC-Server supports network computing that our motion control system TRACK and the IPC-Server can run on different workstations. Here, we are only interested in using the derived data from hand posture input. There are totally 16 data from DataGlove, 10 from finger flexion with 2 for each fingers, and 6 from the palm position and orientation. From section 2, one will find there are 3 flexing DOFs of each finger most significant to our multi-sensor based grasping method, more than those from DataGlove. We solve this problem by the fact that the flexing DOFs on each finger are not independent to move, especially the first and second DOFs. By putting these two in a linear function, the number of DOFs is 10 .

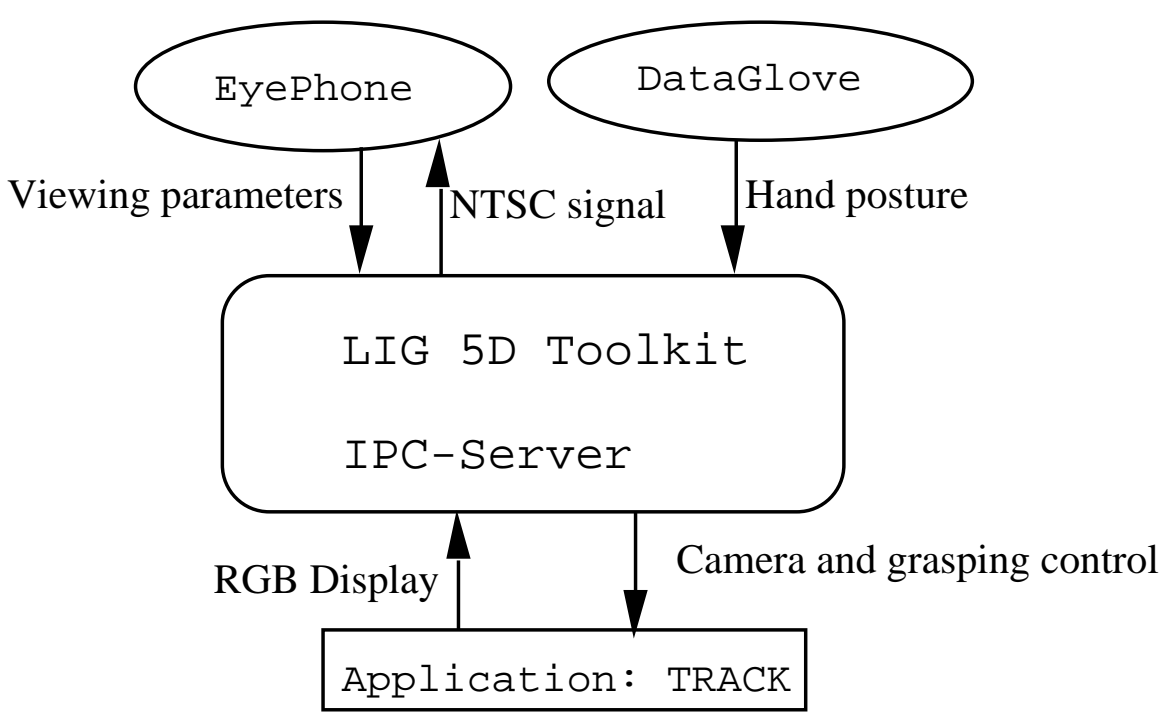

Figure 13. The framework of interactive grasping with DataGlove 
The 10 data from DataGlove finger bending are ranged from 0 to 90 degree, but the respective DOFs of the hand model have different range as shown in Table 3. Before using the data from DataGlove, we should make data mapping.

\begin{tabular}{|llll|l||l|l|l|}
\hline r_pinky_fl1 & r_ring_fl1 & r_middle_fl1 & r_index_fl1 & $(-110,30)$ \\
\hline r_pinky_fl2 & r_ring_fl2 & r_middle_fl2 & r_index_fl2 & $(-110,0)$ & r_thumb_fl1 & $(-35,10)$ \\
\hline r_pinky_fl3 & r_ring_fl3 & r_middle_fl3 & r_index_fl3 & $(-90,0)$ & r_thumb_fl2 & $(-60,0)$ \\
\hline
\end{tabular}

Table 3. The value range (degree) of DOFs controlled by DataGlove

At the beginning of interactive grasping, only the hand center sensor is active. The six palm values from DataGlove are used to move it toward the object. Inverse kinematics is used to update the arm postures from hand center movement. After the sensor is activated, the hand is close enough to the object final frame. The hand center sensor is deactivated and multi-sensors on hand are now used, to detect sensor object collision. The following process is similar to the multi-sensor method discussed before. The major difference is that the grasping strategy is defined interactively. One example is shown in Figure 14.

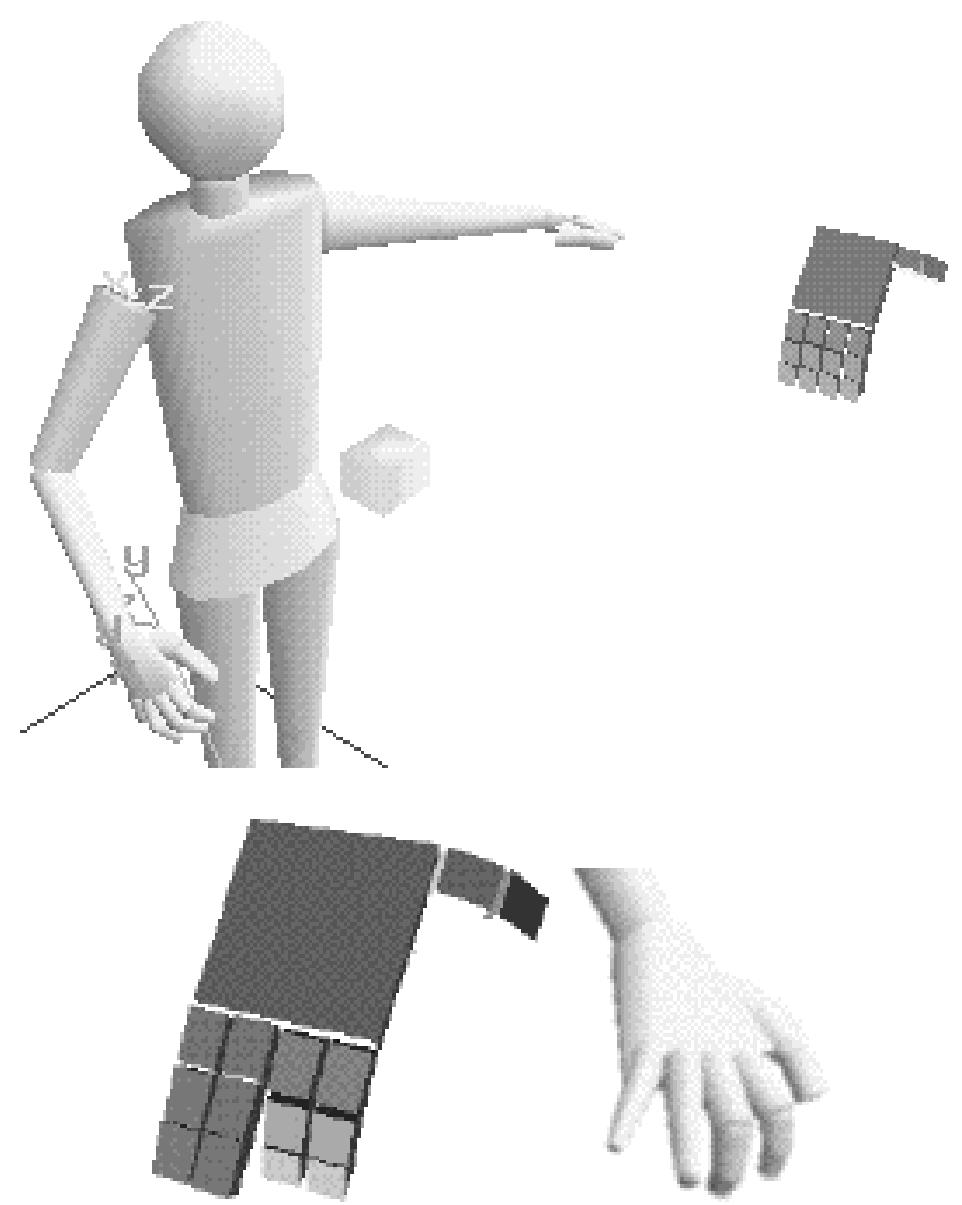

Figure 14. 3D interactive grasping with DataGlove 


\section{Results}

All the grasping functions mentioned above have been implemented and integrated into the TRACK system (Boulic et al. 1994b). The examples shown in this section were performed on SGI Indigo 2 in real time.

The first example shows an actor using two hands for grasping a cube and a cylinder. The strategies are different according to the types of the objects (Figure 15).

The second example shows two actors grasping a frustum. The first actor uses two hands to grasp the thick part, while the second only uses one hand. The three successive frames are shown in Figure 16.

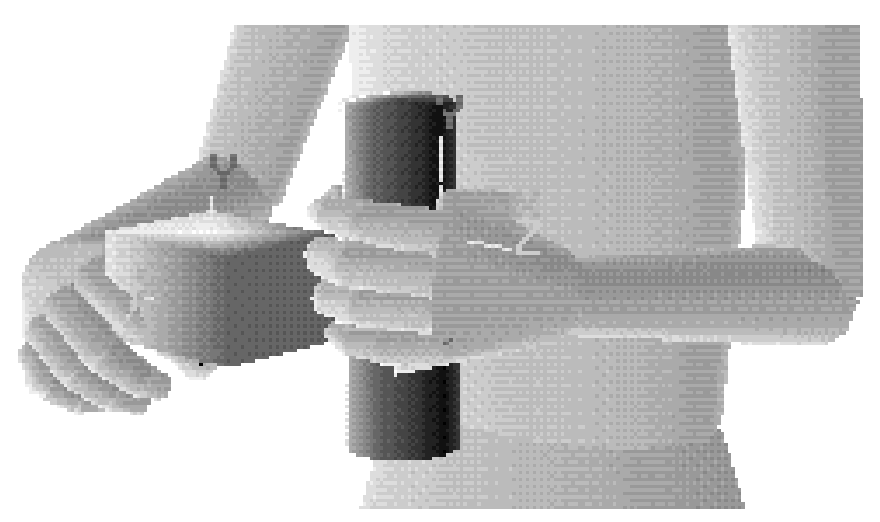

Figure 15. Grasp different objects with two hands
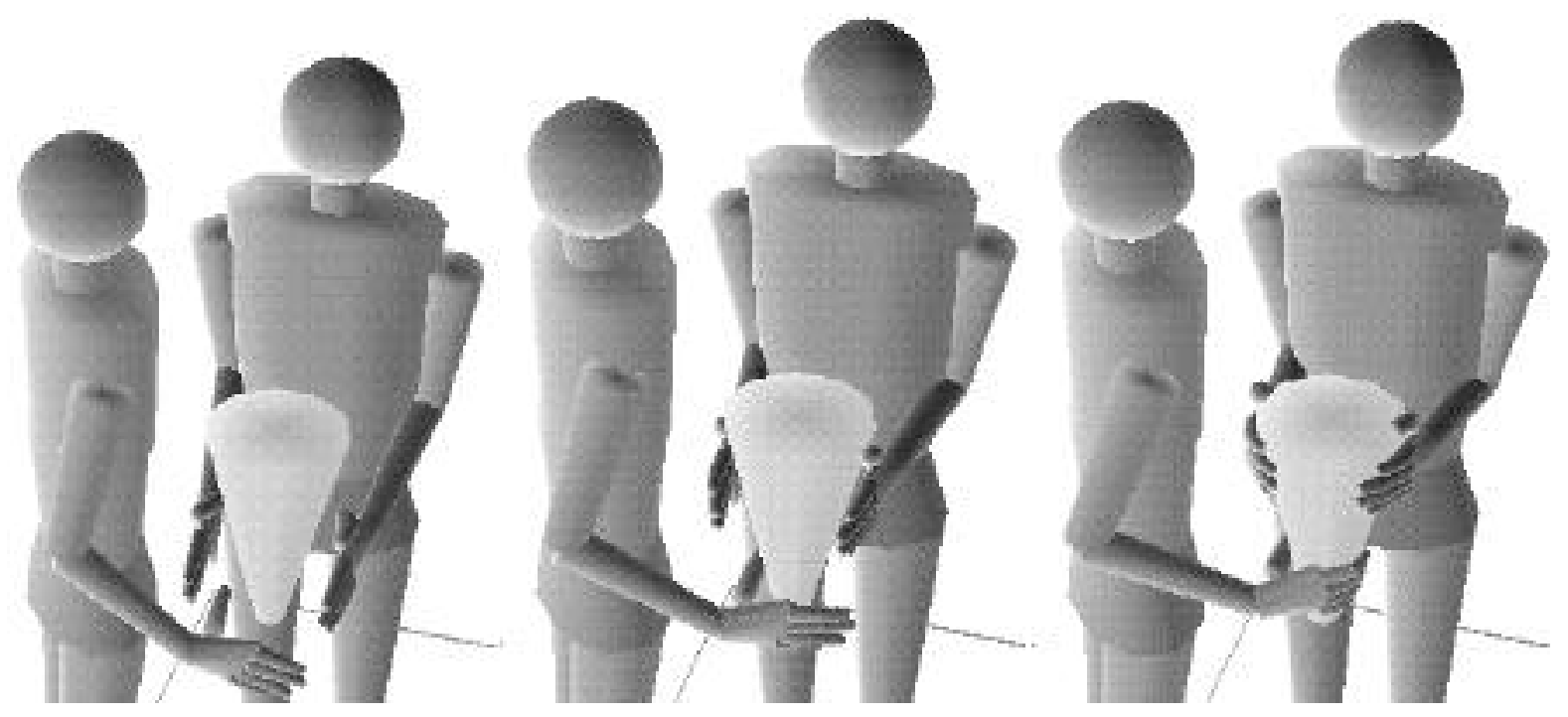

Figure 16. Two actors grasp a frustum at different positions with different ways

In Figure 17, we extend the grasping method to interactions between two actors. Actor hand shaking is shown in Figure 17, the first actor puts his right hand forward and keeps still, then the second grasps the palm of the first actor, that is frustum grasping. After grasping, the first actor closes fingers with sensor object detection to closely hold other's hand.

In Figure 18, one actor uses two hands to grasp a head modeled with a triangle mesh. It demonstrates the generality of the multi-sensor grasping method. To add a new model of object, the only thing is to elaborate its collision algorithm with a sphere sensor. 


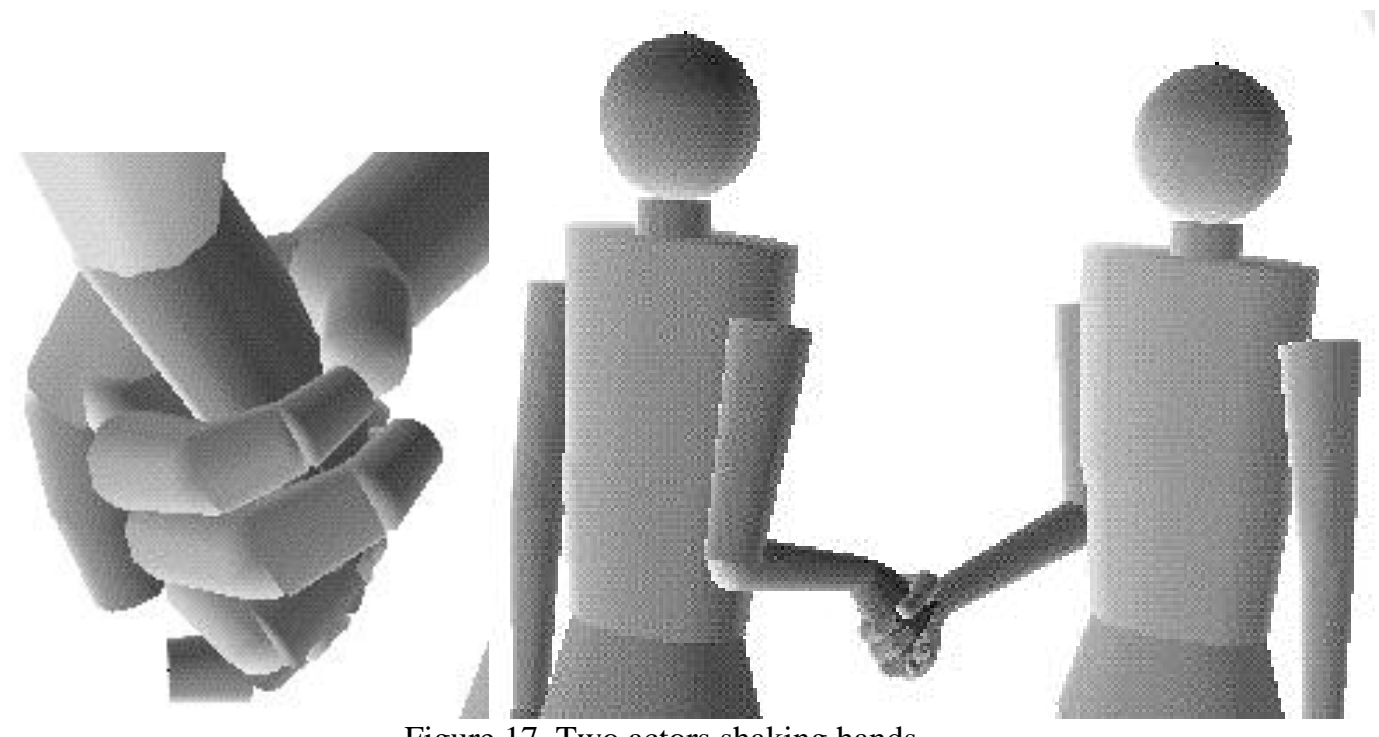

Figure 17. Two actors shaking hands

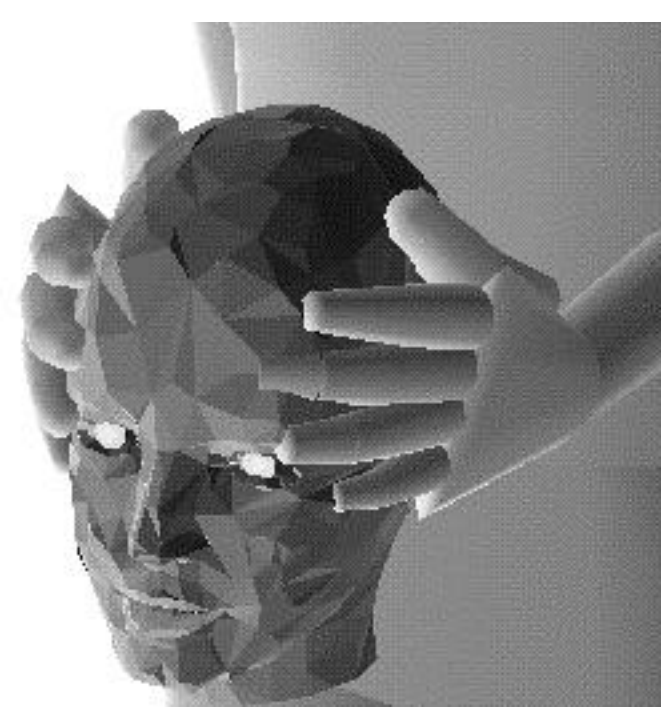

Figure 18. Grasp a more complicated object

In Figures 19, 20 and 21, we show two actors modeled with solid primitives and triangle meshes holding each other. Using traditional key framing, it is very difficult to animate this kind of behavior, but it is automatic animated after the target parts of body are selected. With a more sophisticated reasoning method, even this selection will be automatic in future. 

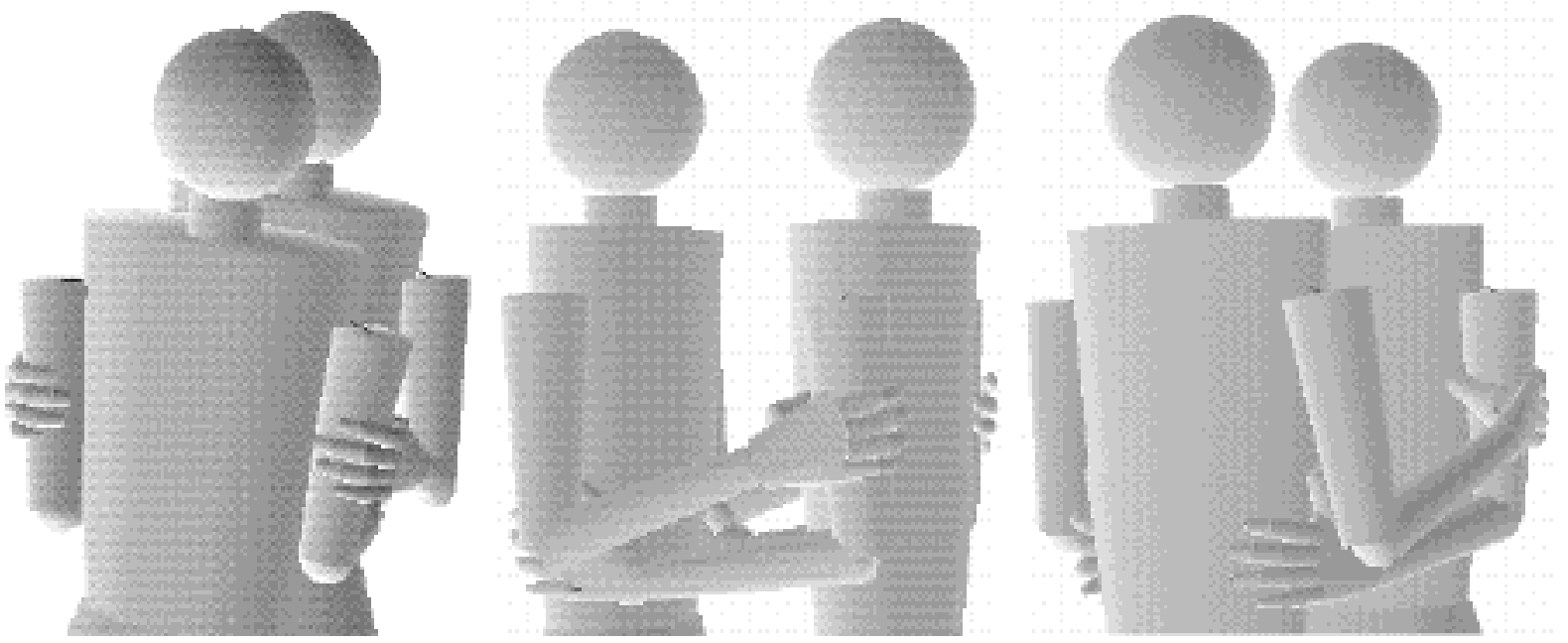

Figure 19. Interaction between two actors modeled by solid primitives
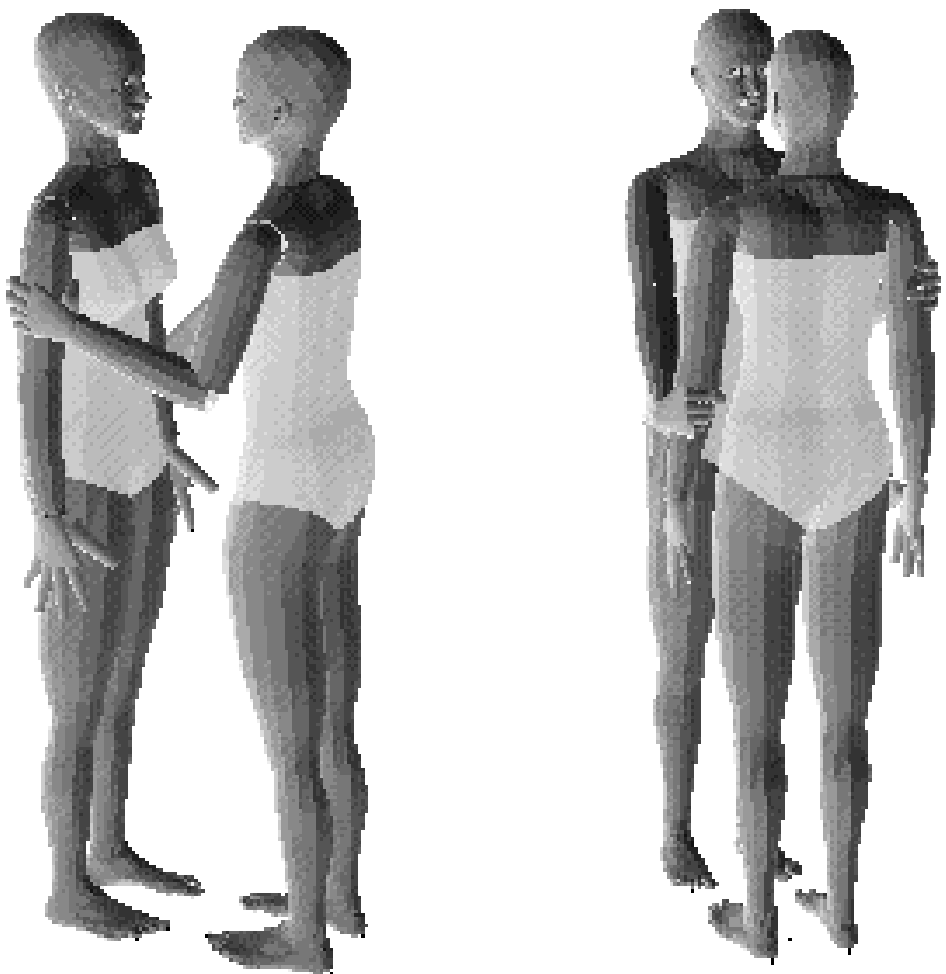

Figure 20. Interaction between two actors modeled by triangle meshes 

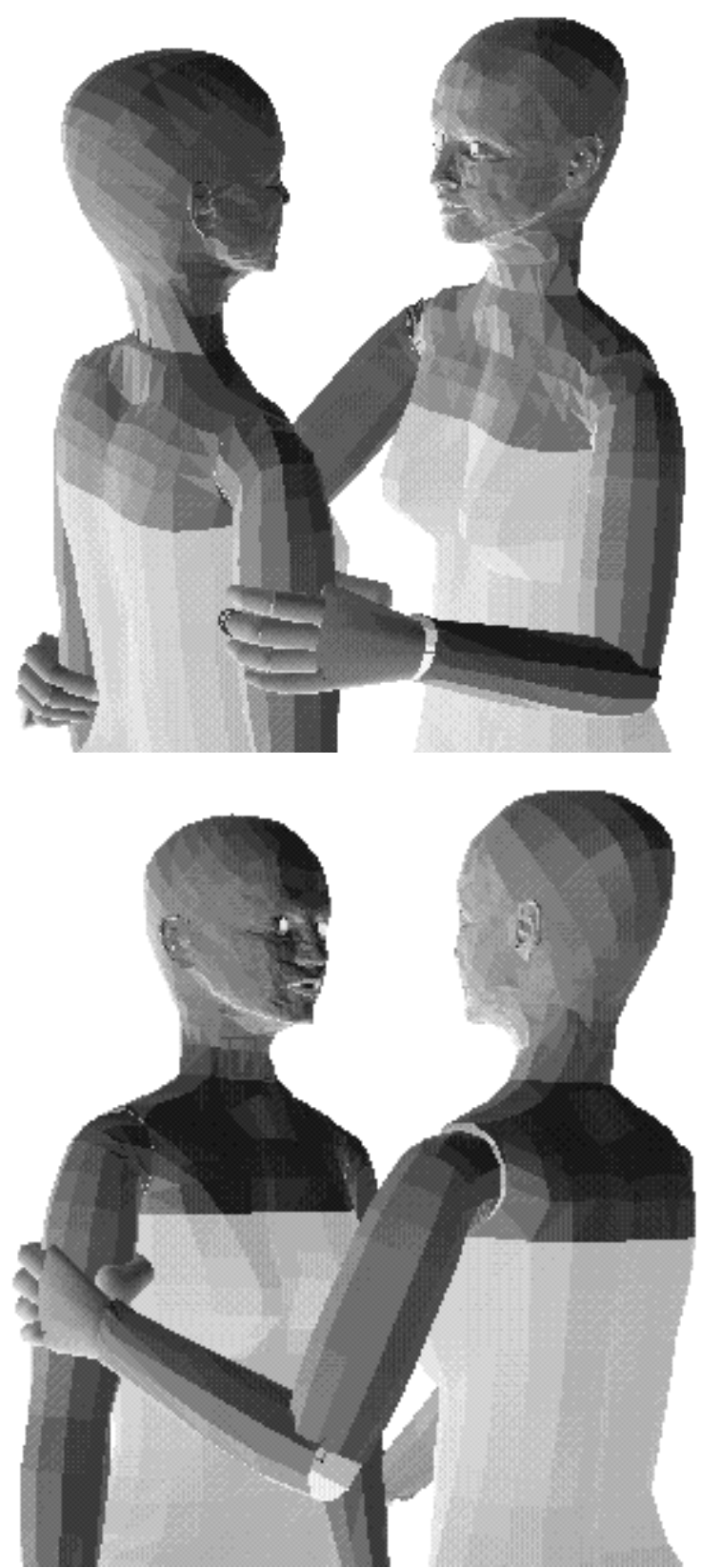

Figure 21. Interaction between two actors modeled by triangle meshes Colour Plate 1. Three frames of holding a baby

\section{Implementation}

The software has been implemented in ANSI C on SGI Indigo 2. As it has been integrated into the TRACK system, the resulting motion can be sampled to produce sequence for refinement or sequence manipulation in TRACK. The modeling of multiple actors is supported by the newly implemented libraries Scenelib and Bodylib (Boulic et al. 94a).

\section{Conclusion}

The heuristic grasping decision and multi-sensor based approach is very efficient and general for grasping motion control. With a group of polynomials based on EulerLagrange equation, the resulting real-time motion is more realistic than linear interpolation. 3D interaction and extension to multiple actors are interesting directions. 
Based on our research in the area of synthetic vision (Renault et al. 1990; Noser et al. 1995), we are now working in vision-based grasping. It will allow the actor to use his visual perception to select the grasping procedure.

\section{Acknowledgments}

We thank our colleague Tom Molet for the implementation of Scenelib and Bodylib for the scene modeling and the generation of multiple actors, J. F. Chavannes, Ch. Currat, and H. Degaudenzi, students in EPFL, for their projects in our lab. Our work is based on the prototype developed by Ramon Mas during his visit to our lab. The research was supported by the Swiss National Science Research Foundation, the Federal Office for Education and Science, and is part of the Esprit Project HUMANOID.

\section{References}

Boulic R., Capin T., Huang Z., Molet T., Shen J., Kalra P., Moccozet L., MagnenatThalmann N., and Thalmann D., (1994a) ESPRIT Project 6709 HUMANOID Report.

Boulic R., Huang Z., Magnenat-Thalmann N., Thalmann D. (1994b) Goal-Oriented Design and Correction of Articulated Figure Motion with the TRACK System , Comput. \& Graphics, Vol. 18, No. 4, pp. 443-452.

Bullock D. and Grossberg S., Neural dynamics of planned-arm movements: emergent invariants and speed accuracy properties during trajectory formation, in Neural Networks and Natural Intelligence, ed. S. Grossberg, MIT Press, 1988.

Espiau B. and Boulic R. (1985) Collision avoidance for redondants robots with proximity sensors, Proc. of Third International Symposium of Robotics Research, Gouvieux, October.

Gobbetti E., Balaguer J.F., and Thalmann D. (1993) VB2: An Architecture for Interaction in Synthetic Worlds, in Proceedings of the ACM Symposium on User Interface Software and Technology, November 1993, pp 167-178.

Kunii TL, Tsuchida Y., Matsuda H., Shirahama M., and Miura S. (1993) A model of hands and arms based on manifold mappings, Proceedings of CGI'93, pp.381-398.

Magnenat-Thalmann N., Laperrière R., and Thalmann D. (1988) Joint-dependent local deformations for hand animation and object grasping, Proceedings of Graphics Interface '88, pp.26-33.

Mas S. R. and Thalmann D. (1994) A Hand Control and Automatic Grasping System for Synthetic Actors, Proceedings of Eurographic'94, pp.167-178.

Noser H., Renault O., Thalmann D., Magnenat Thalmann N. (1995) Navigation for Digital Actors based on Synthetic Vision, Memory and Learning, Computers and Graphics, Pergamon Press, Vol.19, No1, pp.7-19.

Philips C. B., Zhao J., Badler N. I. (1990) Interactive Real-Time Articulated Figure Manipulation Using Multiple Kinematic Constraints, Computer Graphics 24 (2), pp.245-250.

Renault O., Magnenat-Thalmann N.,Thalmann D. (1990) A Vision-based Approach to Behavioural Animation, Journal of Visualization and Computer Animation, Vol.1, No1, pp.18-21. 
Rijpkema H and Girard M. (1991) Computer animation of knowledge-based human grasping, Proceedings of Siggraph'91, pp.339-348.

Turner R., Gobbetii E., Balaguer F., Mangili A., Magnenat-Thalmann N., and Thalmann D., (1990) An Object-Oriented Methodology using Dynamic Variables for Animation and Scientific Visualization, Proceedings of CGI'90, pp.317-327.

van de Panne M., and Fiume E. (1993) Sensor-Actuator Network, Computer Graphics, Annual Conference Series, 1993, pp.335-342. 
Zhiyong Huang is a PhD candidate at EPFL, Switzerland. His research interests include computer graphics and animation. He received a BS in 1986 and a MS in 1988 from the Department of Computer Science and Technology, Tsinghua University.

Dr Ronan Boulic is a Senior Researcher at the Computer Graphics Lab from EPFL Lausanne. He graduated from National Institute of Applied Sciences (INSA-Rennes), with Electronical Engineer Degree in 1983. He made his Ph.D. thesis in Computer Sciences (Robotics) at the National Research Institute in Computer Sciences and Automatic Systems (INRIA -Rennes, IRISA) in 1986. He was a postdoctoral invited researcher at the Computer Graphics lab of University of Montreal, Pr D. Thalmann. $\mathrm{He}$ is Member of the Program Committee of EUROGRAPHICS Workshop on Animation and Simulation (since 1993).

Nadia Magnenat Thalmann is full Professor of Computer Science at the University of Geneva, Switzerland and Adjunct Professor at HEC Montreal, Canada. She has served on a variety of goverment advisory boards and program committees in Canada. In 1987, she was nominated woman of the year by the Montreal community in Quebec. She has received several awards, including the 1985 Communications Award from the Government of Quebec, the Moebius Award from the European Community in 1992, and the British Computer Society Award in 1993. Dr. Magnenat Thalmann received a BS in psychology, an MS in biochemistry, and a Ph.D in quantum chemistry and computer graphics from the University of Geneva. She has written and edited several books and research papers in image synthesis and computer animation and was codirector of the computer-generated films Dream Flight, Englantine, Rendez-vous à Montreal, Galaxy Sweetheart, IAD, Flashback, Still Walking and Fashion Show. She has served as a chairperson of Graphics Interface '85, Computer Graphics International (CGI'88), and the annual workshop and film festival in Computer Animation held in Geneva. She is co-editor-in-chief of the Visualization and Computer Animation Journal, associate editor-in-chief of the Visual Computer, editor of the Computational Geometry Journal and the CADDM journal. She is the President of the Computer Graphics Society (CGS).

Daniel Thalmann is currently full Professor and Director of the Computer Graphics Laboratory at the Swiss Federal Institute of Technology in Lausanne, Switzerland. He is also adjunct Professor at the University of Montreal, Canada. He received his diploma in nuclear physics and Ph.D in Computer Science from the University of Geneva. He is coeditor-in-chief of the Journal of Visualization and Computer Animation, member of the editorial board of the Visual Computer, the CADDM Journal (China Engineering Society) and Computer Graphics (Russia). He is cochair of the EUROGRAPHICS Working Group on Computer Simulation and Animation and member of the Executive Board of the Computer Graphics Society. Daniel Thalmann was member of numerous Program Committees, Program Chair of several conferences and chair of the Computer Graphics International '93 Conference. Daniel Thalmann's research interests include 3D computer animation, image synthesis, virtual reality and scientific visualization. He has published more than 150 papers in these areas, is coeditor of 20 books, and coauthor of several books including: Computer Animation: Theory and Practice and Image Synthesis: Theory and Practice. He is also codirector of several computer-generated films with synthetic actors. 